\title{
Satisfação com a vida, experiência odontológica e autopercepção da saúde bucal entre idosos
}

\author{
Satisfaction with life, dental experience \\ and self-perception of oral health among the elderly
}

\author{
Lilian Rigo ${ }^{1}$ \\ Kenny Basso ${ }^{2}$ \\ Jandir Pauli ${ }^{2}$ \\ Graziela Oro Cericato ${ }^{1}$ \\ Luiz Renato Paranhos ${ }^{3}$ \\ Raissa Rigo Garbin ${ }^{4}$
}

${ }^{1}$ Escola de Odontologia, Faculdade Meridional (IMED). R. Senador Pinheiro 304, Cruzeiro. 99070-220 Passo Fundo RS Brasil. lilianrigo@via-rs.net ${ }^{2}$ Escola de Administração, Faculdade Meridional (IMED).

${ }^{3}$ Universidade Federal de Sergipe.

${ }^{4}$ Faculdade de Medicina, Universidade de Passo Fundo.

\begin{abstract}
The scope of this article is to analyze the relationship between satisfaction with quality of life, self-perception of oral health and experience with dental surgeons. The study is cross-sectional epidemiological in structure with a sample of 326 elderly individuals over 60 years of age living in a city in the north of the State of Rio Grande do Sul, Brazil. The instrument for data collection was a self-administered questionnaire with queries relating to self-perception in oral health (OHIP - Oral Health Impact Profile), Quality of Life Satisfaction scale and sociodemographic issues. The findings showed that the elderly with higher levels of quality of life satisfaction manifested an enhanced perception of their own oral health as well as a better perceived image of dental surgeons and less anxiety about their experiences with the dentist. It was proven that both the self-perception that the elderly have about oral health as well as their experience with dentists is associated with the quality of life satisfaction of the elderly. The results have important implications for decision-makers and formulators of public policy. Key words Quality of life, Oral health, Elderly, Self-perception
\end{abstract}

Resumo $O$ objetivo deste artigo é analisar a relação existente entre a satisfação com a vida $e$ a autopercep̧ção em saúde bucal e a experiência com cirurgiões-dentistas. A pesquisa tem um delineamento epidemiológico transversal, com uma amostra de 326 idosos acima de 60 anos de idade, moradores de um município do norte do Rio Grande do Sul, Brasil. Para o instrumento de coleta de dados foi utilizado um questionário autoaplicativo com questões referentes à autopercepção em saúde bucal (OHIP - Perfil de Impacto na Saúde Oral), Escala de Satisfação com a Vida e dados sociodemográficos. Os resultados mostraram que idosos com maiores níveis de satisfação com a vida apresentaram uma melhor percepção de sua própria saúde bucal, bem como possuem uma melhor imagem percebida dos cirurgiõesdentistas e sentem menos ansiedade em relação à experiência com o dentista. Comprova-se que tanto a autopercepção que os idosos possuem da saúde bucal quanto a experiência com cirurgiõesdentistas possuem uma associação com a satisfação com a vida. Os resultados possuem implicações importantes para tomadores de decisões $e$ formuladores de políticas públicas.

Palavras-chave Qualidade de vida, Saúde bucal, Idoso, Autopercepção 


\section{Introdução}

O Brasil vivenciou um aumento expressivo no número de idosos nas últimas décadas, o que influenciou novos estudos focados nesta faixa etária, englobando pesquisas de envelhecimento e qualidade de vida desta população. $\mathrm{O}$ incremento na expectativa e na qualidade de vida dos idosos está relacionado tanto à evolução da tecnologia e da medicina, quanto à convivência destes em grupos, incluindo atividades físicas e de lazer, aspectos emocionais e comportamentais ${ }^{1}$.

Acrescido a esse fato, o Brasil avançou no campo da saúde pública, somando ações de saúde voltadas para a população idosa e às estratégias de políticas públicas. Esta implementação é importante para assegurar que o processo de desenvolvimento econômico e social ocorra de forma contínua, garantindo um patamar econômico mínimo para a manutenção da dignidade humana, bem como a equidade da partilha dos recursos, direitos e responsabilidades sociais entre os diferentes grupos etários ${ }^{2}$. Por isso, as políticas destinadas aos idosos devem considerar a capacidade funcional, a autonomia, a participação, o cuidado e a autossatisfação ${ }^{3}$.

O levantamento nacional de saúde bucal realizado em 2010 (SB 2010) apontou que mais de 3 milhões da população idosa brasileira entre 65 e 74 anos necessitam de prótese total (nas duas arcadas dentárias), e outros 4 milhões, precisam usar prótese parcial (em uma das arcadas) ${ }^{4}$. Os dados desse levantamento sugerem que quanto mais avançada a faixa etária, maior o índice de pessoas desdentadas, associando-se o envelhecimento da população brasileira com a necessidade do uso de próteses totais.

Na prática clínica diária, é necessário verificar a percepção da saúde bucal e geral que os idosos têm de si, associando esta informação à sua qualidade de vida relatada ${ }^{5}$. Esses indicadores subjetivos preenchem a necessidade de mensurar o impacto que os indivíduos têm na sua condição de saúde ${ }^{6}$. Atualmente, verifica-se que os usuários regulares de serviços odontológicos se declaram mais satisfeitos com suas condições bucais do que aqueles que não frequentam regularmente esses serviços, mostrando que a frequência das visitas ao cirurgião-dentista interfere na qualidade de vida da população ${ }^{7}$. Nesse ponto, cabem os seguintes questionamentos: as pessoas com maiores níveis de satisfação com a vida são, portanto, pessoas com maior frequência e hábitos de tratamentos odontológicos? Maiores níveis de satisfação com a vida estão ligados a menores níveis de problemas odontológicos?
Diante destas questões, e ciente de que as condições de saúde bucal apresentam impacto na vida diária das pessoas, torna-se importante a utilização de indicadores capazes de capturar tal impacto a fim de garantir políticas e estratégias apropriadas, bem como a excelência da Atenção em Saúde e a Qualidade de vida. Nesse sentido, a Escala de Satisfação Com a Vida (ESCV - Satisfaction With Life Scale), criada no início da década de 1980, visa estimar, a partir de uma avaliação global e não por domínios específicos, a satisfação do indivíduo com as suas condições de vida atual em relação ao padrão de vida estabelecido por ele como desejável ${ }^{8}$. As questões de autopercepção em saúde bucal utilizam o instrumento OHIP (Perfil de Impacto na Saúde Oral), por ser um instrumento válido e confiável, para a medição detalhada do impacto social das disfunções orais, além de identificar benefícios potenciais para a tomada de decisão clínica e em pesquisa.

Assim, o objetivo do presente estudo é analisar a relação existente entre a satisfação com a vida e a autopercepção em saúde bucal e experiência com cirurgiões-dentistas. Diante deste objetivo, e baseado no exposto, as hipóteses sugerem que idosos com maiores níveis de satisfação com a vida irão apresentar uma percepção de saúde bucal mais positiva que aqueles com menor. Além disso, também se supõe que por apresentarem uma melhor percepção de sua saúde bucal, os indivíduos mais satisfeitos com a vida irão apresentar um comportamento de menor frequência e menor experiência com cirurgiõesdentistas.

\section{Metodologia}

A presente pesquisa é um estudo epidemiológico de corte transversal, incluindo idosos acima de 60 anos de idade, moradores do município de Passo Fundo, Rio Grande do Sul, Brasil.

A amostra foi constituída por 326 idosos e o tamanho amostral foi calculado tendo como base um total de 21.886 idosos acima de 60 anos de idade existentes na área urbana do município. Para o cálculo do tamanho amostral, de modo que este fosse representativo da população, o coeficiente de confiança de $95 \%$, a margem de erro de $5,4 \%$ e a probabilidade dos agravos de $50 \%$ foi utilizada. A amostragem aleatória do estudo foi estratificada considerando as cinco praças localizadas nos diferentes locais/bairros da cidade (com base na população estimada do bairro, de acordo com dados fornecidos pela Prefeitura Municipal 
de Passo Fundo - RS), obtendo 20\% dos indivíduos de cada local. Além disso, os entrevistadores abordaram somente sujeitos que possuiam os critérios estabelecidos: ser morador do bairro onde foi realizada a coleta dos dados, possuir idade acima de 60 anos e possuir alfabetização suficiente para preenchimento do questionário autoaplicativo. Os locais estabelecidos para a coleta foram as praças públicas do município de Passo Fundo, sendo elas: Praça Marechal Floriano, Praça da Mãe Preta, Praça Professor Ernesto Tochetto, Praça Tamandaré e Praça Capitão Jovino.

A coleta de dados realizada no município ocorreu no mês de setembro de 2012, após aprovação do Comitê de Ética em Pesquisa da Faculdade Meridional de Passo Fundo, RS. O instrumento de coleta de dados foi um questionário autoaplicável destinado aos idosos, contendo questões referentes à satisfação com a vida, autopercepção em saúde bucal e quanto ao tratamento odontológico realizado e dados sociodemográficos. Assim durante a coleta de dados, os entrevistadores apresentavam o instrumento aos participantes do estudo para que lessem e respondessem. Para tanto, o instrumento continha todas as instruções e também todas as escalas utilizadas. Após, os participantes devolviam o instrumento aos pesquisadores, que acompanhavam toda a coleta de dados para redimir quaisquer dúvidas dos participantes.

As questões de autopercepção em saúde bucal foram retiradas do instrumento OHIP (Perfil de Impacto na Saúde Oral). O OHIP é um instrumento que avalia a qualidade de vida relacionada à saúde bucal, contendo questões de domínio físico, psicológico e social ${ }^{9}$. Este instrumento foi utilizado no contexto de idosos por Martins et al. ${ }^{10}$ e Bianco et al. ${ }^{11}$.

Os dados demográficos utilizados foram renda, gênero, estado civil, idade e bairro onde reside. O questionário aplicado com questões referentes à experiência com cirurgiões-dentistas, foi obtido a partir de questões simples relacionadas a ir a consulta odontológica, ter desistido da consulta, representação social do dentista e sensação em ir ao dentista. Tais perguntas foram desenvolvidas pelos pesquisadores e validadas com outros colegas da área e pré-testadas com sujeitos da mesma população do estudo, mas que não compuseram a amostra final. Enquanto a satisfação de vida $(\alpha=0,806)$ foi verificada por escala retirada do estudo de Diener et al. ${ }^{12}$. A "Escala de Satisfação Com a Vida” (ESCV) propõe-se a avaliar o componente cognitivo do bem estar subjetivo, ou seja, a satisfação com a vida. Por meio de uma avaliação global, e não de aspectos da vida específicos, o próprio respondente pode eleger o que é fundamental para sua satisfação com a vida. A escala tem sido amplamente utilizada em diversas populações de diferentes culturas no mundo inteiro. A ESCV é um instrumento autorrespondido de cinco itens. Para análise final, após o teste de confiabilidade da escala, os itens foram transformados, através da média, em uma nova variável, denominada satisfação com vida, para que representasse o construto.

Após a coleta, foi construído um banco de dados para o armazenamento das informações e transportados para o SPSS 17.0 para realização da análise estatística. As análises foram descritivas e inferenciais, a fim de verificar as relações entre as variáveis. Para a análise dos dados e verificação da relação entre o nível de satisfação com a vida e as questões de autopercepção em saúde bucal e com experiência com cirurgiõesdentistas, optou-se por dividir a satisfação com vida em dois extremos: baixa satisfação e alta satisfação, delimitadas pela mediana da distribuição (4,40 em uma escala de 5 pontos). Face a não normalidade dos dados (verificada por meio do teste de Kolmogorov-Smirnov Z; p $<0,05)$, testes não paramétricos como Mann-Whitney $U$ e Qui-Quadrado foram utilizados para verificar as associações. Em cada teste, o valor crítico de significância ( $p$-value) considerado foi 0,05 .

\section{Resultados e discussão}

Para a apresentação da discussão e dos resultados, e consequentemente para o alcance dos objetivos, todas as variáveis utilizadas tiveram sua comparação feita entre indivíduos com baixa e alta satisfação com a vida, conforme descrito no método do estudo.

Dos 326 idosos da amostra, 53,7\% eram do gênero feminino e $46,3 \%$ do gênero masculino. Com relação à faixa etária, 11,3\% com 60 anos, $27,0 \%$ de 61 a 65 anos, 25,5\% de 66 a 70 anos, $18,4 \%$ de 71 a 75 anos, $8,6 \%$ de 76 a 80 anos, $6,4 \%$ de 81 a 85 anos, $2,8 \%$ com 86 anos ou mais. Dos entrevistados, $43,6 \%$ possuem renda familiar de até R\$1.244, 32,5\% entre R\$1.244,00 e $2.488,00,19,3 \%$ entre $\mathrm{R} \$ 2.488,00$ e $\mathrm{R} \$ 6.220,00$, $3,7 \%$ entre $\mathrm{R} \$ 6.220,00$ e $12.440,00$ e $0,9 \%$ acima de R\$12.440,00. Ainda, 7,4\% declararam ser solteiros, $53,7 \%$ casados, $27,9 \%$ viúvos, $2,8 \%$ possuem união estável e 8,3\% são divorciados.

Para fins de comparação sociodemográfica, na Tabela 1 está apresentado o cruzamento das 
variáveis sociodemográficas com a satisfação com a vida.

Na análise da Tabela 1, percebe-se que não há uma associação significativa entre a faixa etária, a renda familiar e o estado civil dos entrevistados com a satisfação com a vida $(\mathrm{p}>0,10)$. No entanto, o gênero dos entrevistados parece estar associado com a satisfação com a vida, sendo que no grupo de alta satisfação a proporção de indivíduos do gênero feminino $(59,6 \%)$ é representativamente maior do que a quantidade de sujeitos do gênero masculino (40,4\%), o que não ocorre no grupo de baixa satisfação, no qual a proporção dos dois gêneros é muito semelhante (Feminino $=49,2 \%$ vs Masculino $=50,8 \%)$. Embora esta associação ocorra, não é possível inferir que o gênero tenha influência na satisfação com a vida. No entanto, futuros estudos podem verificar com maior profundidade a possível associação e suas causas, uma vez que este não é o propósito deste trabalho. Em estudo com população de 365 idosos da cidade de São Paulo em relação à satisfação com a vida, 51,5\% se disseram muito satisfeitos. Os valores correspondentes aos escores mais baixos ocorreram nas variáveis aprendizado escolar e situação financeira ${ }^{13}$. Resultado semelhante foi encontrado em um estudo realizado com cuidadores e não cuidadores, em que a baixa satisfação com a vida de idoso foi associada a insônia e a sintomas depressivos ${ }^{14}$.

Outro estudo, realizado em um pequeno município do interior paulista, o objetivo foi conhecer o grau de satisfação de 322 idosos com a qualidade de vida e suas associações com características sociodemográficas e de morbidade. Os autores concluíram que a satisfação do idoso com sua qualidade de vida está associada principalmente a aspectos emocionais relacionados à motivação para viver e ao conforto mínimo para dignidade da sobrevivência ${ }^{15}$.

Após verificar no presente estudo que apenas a variável gênero esteve associada à satisfação com a vida, procurou-se verificar as relações entre a autopercepção em saúde bucal e a satisfação com a vida. Neste sentido, a Tabela 2 apresenta a ligação da variável satisfação com a vida (classificada em dois grupos: baixa e alta) com os indicadores que compõe o instrumento OHIP utilizado neste estudo.

Tabela 1. Relação entre a Satisfação com a Vida e as características sociodemográficas da amostra.

\begin{tabular}{|c|c|c|c|c|}
\hline \multirow[b]{2}{*}{ Variáveis/Categorias } & \multicolumn{3}{|c|}{ Frequência (\%) } & \multirow[b]{2}{*}{ Diferença } \\
\hline & Baixa Satisfação & Alta Satisfação & Total & \\
\hline \multicolumn{4}{|l|}{ Faixa etária } & \multirow{8}{*}{$\chi^{2}=6,229 ; \mathrm{gl}=6 ; \mathrm{p}=0,39$} \\
\hline Até 60 anos & 8,6 & 14,9 & 11,3 & \\
\hline de 61 a 65 anos & 29,2 & 24,1 & 27,0 & \\
\hline de 66 a 70 anos & 25,9 & 24,8 & 25,5 & \\
\hline de 71 a 75 anos & 16,8 & 20,6 & 18,4 & \\
\hline de 76 a 80 anos & 9,7 & 7,1 & 8,6 & \\
\hline de 81 a 85 anos & 7,6 & 5,0 & 6,4 & \\
\hline 86 anos ou mais & 2,2 & 3,5 & 2,8 & \\
\hline \multicolumn{4}{|l|}{ Renda familiar } & \multirow{6}{*}{$\chi^{2}=3,966 ; \mathrm{gl}=4 ; \mathrm{p}=0,41$} \\
\hline Até R\$ 1.244 & 45,9 & 40,4 & 43,6 & \\
\hline de $R \$ 1.244$ a $R \$ 2.488$ & 31,9 & 33,3 & 32,5 & \\
\hline de $R \$ 2.488$ a $R \$ 6.220$ & 19,5 & 19,1 & 19,3 & \\
\hline de $\mathrm{R} \$ 6.220$ a $\mathrm{R} \$ 12.440$ & 2,2 & 5,7 & 3,7 & \\
\hline Acima de $\mathrm{R} \$ 12.440$ & 0,5 & 1,4 & 0,9 & \\
\hline \multicolumn{4}{|l|}{ Estado civil } & \multirow{6}{*}{$\chi^{2}=5,421 ; g l=4 ; p=0,24$} \\
\hline Solteiro & 9,7 & 4,3 & 7,4 & \\
\hline Casado & 55,1 & 51,8 & 53,7 & \\
\hline Viúvo & 24,9 & 31,9 & 27,9 & \\
\hline União estável & 2,2 & 3,5 & 2,8 & \\
\hline Divorciado & 8,1 & 8,5 & 8,3 & \\
\hline \multicolumn{4}{|l|}{ Gênero } & \multirow{4}{*}{$\chi^{2}=3,471 ; g l=1 ; p=0,06^{*}$} \\
\hline Masculino & 50,8 & 40,4 & 46,3 & \\
\hline Feminino & 49,2 & 59,6 & 53,7 & \\
\hline $\mathrm{N}$ & 185 & 141 & 326 & \\
\hline
\end{tabular}

*Associação estatisticamente significante ao teste do qui-quadrado. 
Tabela 2. Relação entre a Satisfação com a vida e a autopercepção em saúde bucal (OHIP- Perfil de Impacto na Saúde Oral).

\begin{tabular}{|c|c|c|c|c|c|c|}
\hline \multirow[b]{2}{*}{ Variáveis } & \multicolumn{2}{|c|}{ Baixa Satisfação } & \multicolumn{2}{|c|}{ Alta Satisfação } & \multirow[b]{2}{*}{$\mathbf{Z}^{*}$} & \multirow[b]{2}{*}{$\mathbf{p}$} \\
\hline & Média & $\sigma$ & Média & $\sigma$ & & \\
\hline $\begin{array}{l}\text { Você teve problemas para falar alguma palavra por } \\
\text { causa de problemas com seus dentes, sua boca ou } \\
\text { gengiva? }\end{array}$ & 2,63 & 1,62 & 2,13 & 1,54 & $-2,91$ & $<0,01$ \\
\hline $\begin{array}{l}\text { Você sentiu que o sabor dos alimentos ficou pior por } \\
\text { causa de problemas com seus dentes, sua boca ou } \\
\text { gengiva? }\end{array}$ & 2,77 & 1,54 & 2,41 & 1,64 & $-2,19$ & $<0,05$ \\
\hline Você sentiu dores em sua boca ou nos seus dentes? & 3,54 & 1,32 & 3,67 & 1,32 & $-1,03$ & $=0,30$ \\
\hline $\begin{array}{l}\text { Você se sentiu incomodado ao comer algum alimento } \\
\text { por causa de problemas com seus dentes, sua boca ou } \\
\text { gengiva? }\end{array}$ & 3,29 & 1,40 & 3,05 & 1,56 & $-1,22$ & $=0,20$ \\
\hline $\begin{array}{l}\text { Você ficou preocupado por causa de problemas com } \\
\text { seus dentes, sua boca ou gengiva? }\end{array}$ & 3,61 & 1,49 & 3,37 & 1,65 & $-1,05$ & $=0,29$ \\
\hline $\begin{array}{l}\text { Você sentiu-se estressado por causa de problemas com } \\
\text { seus dentes, sua boca ou gengiva? }\end{array}$ & 2,99 & 1,52 & 2,55 & 1,73 & $-2,51$ & $<0,01$ \\
\hline $\begin{array}{l}\text { Sua alimentação ficou prejudicada por causa de } \\
\text { problemas com seus dentes, sua boca ou gengiva? }\end{array}$ & 2,85 & 1,52 & 2,58 & 1,66 & $-1,71$ & $=0,08$ \\
\hline $\begin{array}{l}\text { Você teve que parar suas refeições por causa de } \\
\text { problemas com seus dentes, sua boca ou gengiva? }\end{array}$ & 2,51 & 1,46 & 2,02 & 1,40 & $-3,16$ & $<0,01$ \\
\hline $\begin{array}{l}\text { Você encontrou dificuldade para relaxar por causa de } \\
\text { problema com seus dentes, sua boca ou gengiva? }\end{array}$ & 2,66 & 1,49 & 2,26 & 1,57 & $-2,53$ & $<0,01$ \\
\hline $\begin{array}{l}\text { Você sentiu-se envergonhado por causa de problemas } \\
\text { com seus dentes, sua boca ou gengiva? }\end{array}$ & 2,81 & 1,61 & 2,40 & 1,65 & $-2,27$ & $<0,05$ \\
\hline $\begin{array}{l}\text { Você ficou irritado com outra pessoa por causa de } \\
\text { problemas com seus dentes, sua boca ou gengiva? }\end{array}$ & 2,13 & 1,45 & 1,70 & 1,35 & $-3,44$ & $<0,01$ \\
\hline $\begin{array}{l}\text { Você teve dificuldade em realizar suas atividades } \\
\text { diárias por causa de problemas com seus dentes, sua } \\
\text { boca ou gengiva? }\end{array}$ & 2,14 & 1,42 & 1,74 & 1,30 & $-3,07$ & $<0,01$ \\
\hline $\begin{array}{l}\text { Você sentiu que a vida, em geral, ficou pior por causa } \\
\text { de problemas com seus dentes, sua boca ou gengiva? }\end{array}$ & 2,47 & 1,53 & 2,07 & 1,48 & $-2,76$ & $<0,01$ \\
\hline
\end{tabular}

* Teste Mann-Whitney U com nível de significância de 0,05. Obs.: 1- Nunca Muito; 2- Quase nunca; 3- Poucas Vezes; 4- As vezes; 5- Frequentemente.

Por meio do teste Mann-Whitney $U$ foi possível identificar que a frequência com que os indivíduos evidenciaram problemas para falar, devido aos problemas bucais, apresentou diferença significativa entre os grupos de satisfação com a vida. Indivíduos com alta satisfação $(\bar{x}=$ 2,63 ) encontraram tais problemas durante a vida menos frequentemente que aqueles com baixa satisfação $(\bar{x}=2,13)$. Aliado a isto, sujeitos com baixa satisfação afirmam sentirem-se mais envergonhados $(\bar{x}=2,81)$ por causa de problemas com seus dentes, boca e gengiva do que indivíduos com alta satisfação $(\bar{x}=2,40)$.

Relacionado a problemas alimentares, indivíduos com menos satisfação com a vida $(\bar{x}=2,77)$ relataram sentir menos o sabor dos alimentos com maior frequencia do que indivíduos com alta satisfação com a vida $(\bar{x}=2,41)$. Parar uma refeição por causa de problemas dentários também apresentou diferença entre os grupos de satisfação com a vida, estando, portanto, ligada a geração de satisfação com vida. Neste aspecto, indivíduos com baixa satisfação $(\bar{x}=2,51)$ relataram uma frequência maior de ocorrência desta interrupção do que aqueles com alta satisfação $(\bar{x}=2,02)$.

Aliado a estes fatores, o sentimento de estresse devido a problemas bucais permeia com maior frequência indivíduos no grupo de baixa satisfação com a vida $(\bar{x}=2,99)$ em comparação com a frequência de tal sentimento entre aqueles com alta satisfação $(\bar{x}=2,55)$. No mesmo raciocínio, indivíduos com baixa satisfação com a vida $(\bar{x}=$ $2,66)$ salientaram que problemas de relaxamento decorrentes de problemas bucais são mais fre- 
quentes do que o relato por indivíduos no grupo de alta satisfação. Isto por revelar que a dificuldade de relaxamento devido a problemas bucais reduz os níveis de satisfação com a vida em geral. Sentimento de irritação com outras pessoas por causa dos problemas bucais também são mais frequentes em indivíduos com baixa satisfação com a vida $(\bar{x}=2,13)$ do que em indivíduos com alta $(\bar{x}=1,70)$. Neste aspecto, sentimentos negativos parecem emergir mais facilmente em indivíduos com problemas bucais, o que, por consequência, reduz os níveis de satisfação com a vida.

Por fim, de uma forma geral, indivíduos com baixa satisfação com a vida $(\bar{x}=2,14)$ encontram mais dificuldade para realizar as atividades diárias devido a problemas com seus dentes, boca e gengiva do que indivíduos com alta satisfação $(\bar{x}$ $=1,74)$. Além disso, para estes sujeitos, com baixa satisfação $(\bar{x}=2,47)$ há uma maior percepção de que a vida ficou pior por causa dos problemas dentários, do que a percepção encontrada em indivíduos com alta $(\bar{x}=2,07)$. Assim, confirma-se a suposição de que os problemas bucais, de uma forma geral, incorrem em uma redução da satisfação com a vida do indivíduo.

Na mesma linha dos achados deste trabalho, um estudo com amostra aleatória de 561 idosos de 70 anos de idade verificou a qualidade de saúde oral de vida (QVRSB) em relação a diferentes condições bucais crônicas e a percepção da saúde bucal, utilizando o Perfil Oral Health Impact (OHIP-14). Os resultados revelaram três variáveis altamente preditivas de indivíduos com altos escores de OHIP-14: o uso de próteses, presença de problemas de mastigação e descontentamento com a aparência dos dentes ${ }^{16}$. Em outra pesquisa feita somente em mulheres acima de 60 anos avaliando a relação entre autopercepção da saúde bucal percebida e a satisfação global com a vida a partir do índice GOHAI (Índice de Determinação da Saúde Oral Geriátrica) e da Escala de Auto-Avaliação de Cantril, 58,9\% pontuaram como baixa sua saúde bucal e $70,6 \%$ das idosas classificaram como alta e positiva sua satisfação global com a vida. Os dados sugerem que, apesar das idosas relatarem que a qualidade de sua saúde bucal estava insatisfatória, esta não interferiu na sua alta satisfação global com a vida ${ }^{5}$. O objetivo de um estudo com pacientes parcialmente dentados foi verificar se a capacidade de mastigação estava relacionada à QVRSB. A maior capacidade mastigatória foi correlacionada com menores escores de OHIP, indicando melhor QVRSB ${ }^{17}$.

Em outro estudo com amostra de idosos acima de 60 anos, observou-se que a autopercepção da saúde bucal, a partir do índice GOHAI, é alta, tanto nos grupos com quanto nos sem acesso a tratamento odontológico conveniado ${ }^{18}$. A mesma percepção positiva com a saúde bucal e a aparência foram verificadas em pesquisa que analisou os dados de idosos do Levantamento Epidemiológico das Condições de Saúde Bucal da população brasileira (Projeto SB Brasil) ${ }^{19}$.

Para verificar a relação entre a satisfação com a vida e a experiência dos cirurgiões-dentistas, a Tabela 3 apresenta o cruzamento da variável satisfação com a vida (a qual fora dividida entre os indivíduos com baixa e alta satisfação) e os indicadores de experiência com cirurgiões-dentistas.

Os resultados evidenciados na Tabela 3 destacam que há uma associação significativa entre a visita ao dentista e a satisfação com a vida $\left(\chi^{2}=\right.$ $6,899 ; \mathrm{gl}=1 ; \mathrm{p}<0,01)$. Nesta associação, há uma maior proporção de indivíduos que não tiveram experiência com um dentista no grupo de alta satisfação com a vida $(6,4 \%)$, em comparação com o de baixa $(1,1 \%)$, o que pode revelar que os indivíduos não necessariamente associam os cuidados e a saúde bucal com um aumento na satisfação com a vida. Convém ressaltar, no entanto, que é baixa a quantidade absoluta de indivíduos que nunca tiveram uma experiência com um dentista $(3,4 \%)$.

Quando questionados se já haviam desistido de um tratamento dentário com um dentista, $80,1 \%$ dos sujeitos no grupo de alta satisfação elencaram que não desistiram, enquanto que $28,6 \%$ dos sujeitos com baixa satisfação elencaram que já desistiram, evidenciando uma associação marginal entre a continuidade do tratamento dentário e a satisfação com a vida $\left(\chi^{2}=\right.$ $3,311 \mathrm{gl}=1 ; \mathrm{p}=0,06)$. Embora marginal $(\mathrm{p}=$ 0,06 ), a direção da associação entre a continuidade ou desistência do tratamento dentário e a satisfação com a vida revela que iniciar e terminar um tratamento dentário pode estar ligado com a satisfação com a vida, e que, por outro lado, a desistência pode revelar uma frustração, diminuindo com isso a satisfação com a vida.

A associação entre a representação do dentista e a satisfação com a vida $\left(\chi^{2}=12,565 \mathrm{gl}=3\right.$; p $<0,01)$ evidencia, por sua vez, que a quantidade de indivíduos com alta satisfação que associam o dentista à satisfação $(65,2 \%)$ é maior que a quantidade de indivíduos com baixa qualidade de vida que associam o dentista à satisfação (48,1\%). Por outro lado, a quantidade de indivíduos com menor satisfação com a vida que associam o dentista a custo $(30,3 \%)$ é maior que a quantidade de indivíduos com alta satisfação que associam 
Tabela 3. Relação entre a Satisfação com a vida e a experiência com cirurgiões-dentistas.

\begin{tabular}{|c|c|c|c|c|}
\hline \multirow[b]{2}{*}{ Variáveis/Categorias } & \multicolumn{3}{|c|}{ Frequência (\%) } & \multirow[b]{2}{*}{ Diferença } \\
\hline & Baixa Satisfação & Alta Satisfação & Total & \\
\hline \multicolumn{5}{|l|}{ Consulta ao dentista } \\
\hline Sim & 98,9 & 93,6 & 96,6 & $\chi^{2}=6,899 ; \mathrm{gl}=1 ; \mathrm{p}<0,01^{*}$ \\
\hline Não & 1,1 & 6,4 & 3,4 & \\
\hline \multicolumn{5}{|l|}{ Já desistiu de algum } \\
\hline tratamento com um dentista & & & & $\chi^{2}=3,311 \mathrm{gl}=1 ; \mathrm{p}=0,06$ \\
\hline Sim & 28,6 & 19,9 & 24,8 & \\
\hline Não & 71,4 & 80,1 & 75,2 & \\
\hline O que um dentista representa & & & & $\chi^{2}=12,565 \mathrm{gl}=3 ; \mathrm{p}<0,01^{*}$ \\
\hline Medo & 15,1 & 14,9 & 15,0 & \\
\hline Dor & 6,5 & 5,0 & 5,8 & \\
\hline Custo & 30,3 & 14,9 & 23,6 & \\
\hline Satisfação & 48,1 & 65,2 & 55,5 & \\
\hline Como se sente ao ir ao dentista & & & & $\chi^{2}=12,625 \mathrm{gl}=4 ; \mathrm{p}<0,05^{*}$ \\
\hline Relaxado & 30,8 & 48,9 & 38,7 & \\
\hline Um pouco ansioso & 34,6 & 29,1 & 32,2 & \\
\hline Ansioso & 24,3 & 13,5 & 19,6 & \\
\hline Muito ansioso & 7,0 & 5,7 & 6,4 & \\
\hline Sente-se mal & 3,2 & 2,8 & 3,1 & \\
\hline $\mathbf{N}$ & 185 & 141 & 326 & \\
\hline
\end{tabular}

*Estatisticamente significante. Teste qui-quadro com nível de significância de 0,05.

o dentista a esta representação (14,9\%). Estas diferenças podem indicar que quando os indivíduos têm uma representação mais positiva (p. ex. satisfação) do dentista, sua satisfação com a vida como um todo é maior, ao passo que representações negativas (p. ex. custo) podem estar ligadas a baixa na satisfação.

A satisfação com a vida também perpassa os sentimentos do indivíduo ao frequentar um dentista, sendo que esta associação foi encontrada e evidenciada na Tabela $2\left(\chi^{2}=12,625 \mathrm{gl}=4\right.$; $\mathrm{p}<$ $0,05)$. Neste sentido, o percentual de sujeitos com alta satisfação com a vida que afirmou sentir-se relaxado $(48,9 \%)$ foi maior do que o percentual de indivíduos com baixa satisfação sob o mesmo sentimento $(30,8 \%)$. Por outro lado, os sentimentos de ansiedade foram mais presentes no grupo de baixa satisfação com a vida (65,9\% disseram sentirem-se ansiosos) do que no grupo de alta (48,3\% disseram sentirem-se ansiosos). Esta ansiedade sentida pelos indivíduos associada ao ato de frequentar o dentista pode fazer com que eles percebam menos satisfação com a vida.

$\mathrm{Na}$ associação relatada na Tabela 3 , há uma maior proporção de indivíduos que não tiveram experiência com um dentista no grupo de alta satisfação com a vida $(6,4 \%)$, em comparação com o de baixa $(1,1 \%)$, o que pode revelar que os indivíduos não necessariamente associam os cuidados e saúde bucal com um aumento na satisfação com a vida. Resultado curioso também foi apresentado em estudo utilizando o indicador subjetivo Dental Impact on Daily Living (DIDL) na mensuração do impacto dos problemas bucais nas dimensões da qualidade de vida. Identificouse que os indivíduos que utilizavam os serviços por motivo de urgência apresentam maior insatisfação ${ }^{7}$. Foram avaliados os fatores associados com o impacto da saúde bucal na qualidade de vida de 504 idosos brasileiros, incluindo exames clínicos bucais e entrevistas com instrumento Oral Health Impact Profile (OHIP-14) para medir QVRSB. Informações sobre características sociodemográficas, utilização de serviços odontológicos e as medidas subjetivas de saúde foram coletadas. As seguintes variáveis foram associadas com um impacto negativo sobre QVRSB: sexo feminino, classe baixa, até 3 pares de oclusão dos dentes posteriores; pelo menos um dente cariado não tratado; razões curativas para a última consulta odontológica; baixa autopercepção de saúde bucal; e má percepção dental quanto aos cuidados prestados ${ }^{20}$. 


\section{Conclusão}

A partir dos resultados encontrados, comprovase que tanto a autopercepção que os idosos possuem da saúde bucal quanto a experiência com cirurgiões-dentistas possui associação com a satisfação com a vida destes idosos.

Os resultados encontrados possuem implicações importantes para tomadores de decisões e formuladores de políticas públicas.

\section{Referências}

1. Penna FB, Santos FHE. O movimento das emoções na vida dos idosos: um estudo com um grupo da terceira idade. Rev Eletrônica de Enfermagem 2006 [acessado 2009 maio 12];8(1):[17-24]. Disponível em: http:// www.fen.ufg.br/revista/revista8_1/original_02.htm

2. Gaétan L, Balestat G. The Disability Study Expert Group Members. Trends in severe disability among elderly people: assessing the evidence in 12 OECD countries and the future implications [Internet]. Paris: OECD Health Working Papers; 2007. [cited 2012 Aug 15]. Available from: http://www.oecd.org/els/soc/38343783.pdf

3. Veras R. Envelhecimento populacional contemporâneo: demandas, desafios e inovações. Rev Saude Publica 2009; 43(3):548-554.

4. Brasil. Ministério da Saúde (MS). Secretaria de Atenção à Saúde. Departamento de atenção Básica. Projeto SBBrasil 2010: Pesquisa Nacional de Saúde Bucal - Resultados Principais. Brasília: MS; 2014.

5. Silva EFA, Sousa MLR. Autopercepção da saúde bucal e satisfação com a vida em mulheres idosas usuárias de prótese total. Rev Odontologia da Universidade Cidade de São Paulo 2006; 18(1):61-65.

6. Saloto JPS, Miotto MHMB, Barcello LA. Percepção sobre saúde bucal de usuários dos serviços odontológicos do município de Iúna-ES. UFES Rev Odontol 2007; 9(3):31-36.

7. Pandolfi M, Barcellos LA, Miotto MHMB, Góes PSA. Saúde Bucal e Qualidade de Vida de Usuários dos Serviços Odontológicos de Vitória (ES). Pesq Bras Odontoped Clin Integr 2011; 11(3):311-316.

8. Gonçalves DM, Kapczinski F. Transtorno mental, indicadores demográficos e satisfação com a vida. Rev Saude Publica 2008; 42(6):1060-1066.

9. Slade GD, Spencer AJ. Development and evaluation of the Oral Health Impact Profile. Community Dent Health 1994; 11(1):3-11.

10. Martins AMEB, Jones KM, Souza JGS, Pordeus IAL. Association between physical and psychosocial impacts of oral disorders and quality of life among the elderly. Cien Saude Colet 2014; 19(8):3461-3478.

11. Bianco VC, Lopes ES, Borgato MH, Silva PM, Marta SN. O impacto das condições bucais na qualidade de vida de pessoas com cinquenta ou mais anos de vida. Cien Saude Colet 2010; 15(4):2165-2172.

\section{Colaboradores}

L Rigo trabalhou na estruturação do artigo, delineamento do método, elaboração do banco de dados e análise dos resultados. K Basso trabalhou digitação do banco de dados e redação do artigo. J Pauli trabalhou na concepção do projeto e na coleta dos dados. RR Garbin participou da discussão da metodologia e da análise estatística dos dados. GO Cericato e LR Paranhos trabalharam na discussão e redação final do artigo.

12. Diener E, Emmons RA, Larsen RJ, Griffin S. The Satisfaction with Life Scale. J Pers Assess 1985; 49(1):71-75.

13. Joia LC, Ruiz T, Donalisio MR. Condições associadas à satisfação com a vida. Rev Saude Publica 2007; 41(1):131-138.

14. Tomomitsu MRSV, Perracini MR, Neri AL. Fatores associados à satisfação com a vida em idosos cuidadores e não cuidadores. Cien Saude Colet 2014; 19(8):34293440.

15. Ruiz T, Monteiro A, Corrente JE, Colombini Netto M. Avaliação do grau de satisfação dos idosos com a qualidade de vida em um pequeno município do estado de são paulo. Rev APS 2007; 10(1):4-13.

16. Stenman U, Ahlqwist M, Björkelund C, Hakeberg M. Oral health-related quality of life - associations with oral health and conditions in Swedish 70-year-old individuals. Gerodontology 2012; 29(2):440-446.

17. Inukai M, John MT, Igarashi Y, Baba K. Association between perceived chewing ability and oral health-related quality of life in partially dentate patients. Health Qual Life Outcomes 2010 8:118.

18. Silva DS, Sousa MLR, Wada RS. Autopercepção e condições de saúde bucal em uma população de idosos. Cad Saude Publica 2005; 21(4):1251-1259.

19. Martins AMBEL, BarretoII, SM, Silveira MF, Santa -Rosa TTA, Pereira RD. Autopercepção da saúde bucal entre idosos brasileiros. Rev Saude Publica 2010; 44(5):912-922.

20. Ulinski KGB, Nascimento MA, Lima AMC, Benetti AR, Poli-Frederico RC, Fernandes KB, Fracasso ML, Maciel SM. Factors Related to Oral Health-Related Quality of Life of Independent Brazilian Elderly. Independent Brazilian Elderly. Int J Dent 2013; 2013:705047.

Artigo apresentado em 16/09/2014

Aprovado em 02/02/2015

Versão final apresentada em 04/02/2015 